Pacific Journal of Mathematic 


\title{
SOME INEQUALITIES IN CERTAIN NONORIENTABLE RIEMANNIAN MANIFOLDS
}

\author{
P. M. PU
}

1. Introduction. As is well known, the projective plane and the Moebius strip are nonorientable manifolds of dimension two. After introducing a Riemannian metric on each of them, we obtain two 2-dimensional nonorientable Riemannian manifolds. For convenience of reference, let us denote them by $M_{p^{2}}^{2}$ and $M_{m}^{2}$, respectively. Each of these manifolds has an area $A$. Moreover, there exists a family of closed curves, which are not homotopic to zero, on each manifold; and hence the set of the lengths of all these closed curves in consideration has a positive greatest lower bound, $a$. The purpose of this paper is to investigate the relationship between these two geometrical constants, $A$ and $a$. It is found that, in each case, there exists an inequality [1] connecting them, of the form

$$
A \geq k a^{2},
$$

$k$ being a constant depending only on the conformal character of the Riemannian manifold. To establish such inequalities and to determine the corresponding best possible constants are the two central problems in this investigation.

For the time being, the projective plane is used in the following realization: it is given as the unit sphere with identification of diametrically opposite points. We assume further that the metric on $M_{p^{2}}^{2}$ is given by

$$
d s^{2}=g(p) d \rho^{2},
$$

$d \rho^{2}$ being the line element of the unit sphere taken from the embedding Euclidean space; $g(p) \in C_{\omega}, g(p)>0$ for any point $p$ on the manifold. As for the Moebius strip, we assume that it is given by the strip

$$
-\beta<y<\beta,
$$

with identification given by the fundamental group

Received July 24, 1951. The author wishes to express his gratefulness to Professor Charles Loewner for his guidance and encouragement in the preparation of this paper which represents the essential contents of his doctor thesis at Syracuse University.

Pacific J. Math. 2 (1952), 55-71 


$$
\begin{aligned}
& x^{\prime}=x+n(2 \alpha), \\
& y^{\prime}=(-1)^{n} y
\end{aligned}
$$$$
(n=0, \pm 1, \pm 2, \cdots)
$$

We assume further that the metric on $M_{m}^{2}$ is given by

$$
d s^{2}=g(x, y)\left(d x^{2}+d y^{2}\right)
$$

where $g(x, y) \in C_{\omega}$ and $g(x, y)>0$. We shall see later that these assumptions are admissible in our cases.

The main idea of the method for solving these problems is to reduce the general metric, $g(p) d \rho^{2}$, to a simple and special one, $\bar{g} d \rho^{2}$, for which the equality in (1) holds, by an averaging process over a certain continuous group space; this enables us to handle our problems more easily. Let $A_{g}, a_{g}, A_{\bar{g}}, a_{\bar{g}}$ be the geometrical constants defined in terms of the original metric and the simplified metric respectively. Fortunately, this averaging process provides us a means of comparison between $A_{g}$ and $A_{\bar{g}}$ and between $a_{g}$ and $a_{\bar{g}}$; namely, we have

$$
A_{g} \geq A_{g}^{-}
$$

$$
a_{g} \leq a_{g}
$$

A comparison of the equality yielded by the special metric mentioned above with the foregoing inequalities (2) gives us the desired result.

Take, for example, the manifold $M_{p^{2}}^{2}$. Each rotation of a 2-sphere about its center in the ordinary space is actually a conformal mapping of $M_{p^{2}}^{2}$ onto itself. All these rotations form a compact Lie group $G$. Averaging $[g(p)]^{1 / 2}$ over $G$ by the Hurwitz integration,

$$
\int_{G}\left[(g(p))^{1 / 2}\right]^{\sigma} \delta \sigma=h^{1 / 2}
$$

where $\sigma \in G$, and where $\delta \sigma$ is the invariant volume element, we can easily show that $h$ is a constant and that the simplified metric is an elliptic one; this produces the equality

$$
A_{h}=\frac{2}{\pi} a_{h}^{2} .
$$

A combination of (3) with the following inequalities corresponding to (2), 


$$
\begin{aligned}
& A_{g} \geq A_{h}, \\
& a_{g} \leq a_{h},
\end{aligned}
$$

shows that we have, in general,

$$
A \geq \frac{2}{\pi} a^{2}
$$

The same method can be extended, with some restrictions, to the case of $M_{p^{n}}^{n}$, that is, the Riemannian manifold whose underlying topological space is an $n$-dimensional projective space.

In the case of $M_{m}^{2}$, let the rectangle

$$
R:\left\{\begin{array}{l}
-\alpha \leq x<\alpha \\
-\beta<y<\beta
\end{array}\right.
$$

be its fundamental region, as will be explained in $\S 3$. There exists a one-parameter family of conformal mappings of $M_{m}^{2}$ onto itself,

$$
\begin{aligned}
& x^{\prime}=x+c, \\
& y^{\prime}=y,
\end{aligned}
$$

$c$ being real $\bmod (4 \alpha)$. Averaging $[g(x+c, y)]^{1 / 2}$ over the interval $[0,4 \alpha]$ by the formula

$$
\frac{1}{4 \alpha} \int_{0}^{4 a}[g(x+c, y)]^{1 / 2} d c=[\bar{g}(y)]^{1 / 2}
$$

we can see that $[\bar{g}(y)]^{1 / 2}$ is free of $x$ and is an even function on account of the fact that the metric is invariant under the fundamental group $\Gamma$; that is,

$$
g\left[x+n(2 \alpha),(-1)^{n} y\right]=g(x, y) .
$$

A further consideration of the same problem with the metric $g(y)\left(d x^{2}+d y^{2}\right)$, where $g(y)$ is positive and even, leads to a distinguished $g_{0}(y)$ such that $g_{0}(y)\left(d x^{2}+d y^{2}\right)$ plays the same role as the elliptic metric in the case of $M_{p^{2}}^{2}$ or $M_{p^{n}}^{n}$; that is, $g_{0}(y)\left(d x^{2}+d y^{2}\right)$ leads to the equality in (1).

2. Riemannian manifold $M_{p^{2}}^{2}$, whose underlying topological space is a projective plane $P^{2}$. To begin with, let us prove the following general lemma, which will often be used. 
Lemma 1. Let $M_{i}^{2}(i=1,2, \cdots, n)$ be a set of $n$ 2-dimensional Riemannian manifolds smooth of order 1 such that each $M_{i}^{2}$, with the same underlying topological space $T^{2}$, has a metric of the form

$$
d s_{i}^{2}=g_{i}(p) d s^{2},
$$

where $g_{i}(p)>0, g_{i}(p) \in C_{0}$ for $p \in M_{i}^{2}$; and $d s^{2}$ is a Riemannian metric which can be defined locally by

$$
d s^{2}=\sum_{j, k=1}^{2} g_{j k}\left(u_{1}, u_{2}\right) d u^{j} d u^{k}, \quad g_{j k}\left(u_{1}, u_{2}\right) \in C_{0} .
$$

Let $\bar{g}_{n}(p)$ be defined by the formula

$$
\bar{g}_{n}(p)=\left\{\frac{\left[g_{1}(p)\right]^{1 / 2}+\cdots+\left[g_{n}(p)\right]^{1 / 2}}{n}\right\}^{2} .
$$

If the sets of lengths $S_{i}=\int_{C}\left[g_{i}(p)\right]^{1 / 2} d s(i=1,2, \cdots, n)$ of a family $F$ of curves $C$ on $T^{2}$ have the same nonnegative greatest lower bound,

$$
a_{g_{1}}=a_{g_{2}}=\cdots=a_{g_{n}},
$$

and the areas $A_{g_{i}}$ of $M_{i}^{2}$ have the same value,

$$
A_{g_{1}}=A_{g_{2}}=\cdots=A_{g_{n}},
$$

then we have

$$
A_{g_{n}} \leq A_{g_{1}}=\cdots=A_{g_{n}}
$$

and

$$
a_{g_{n}}^{-} \geq a_{g_{1}}=\cdots=a_{g_{n}} \text {. }
$$

Proof. By the definition of area and that of $\bar{g}_{n}$, we have

$$
A_{\bar{g}_{n}}=\iint \bar{g}_{n}(p) d \omega=\iint \frac{\left[\left(g_{1}\right)^{1 / 2}+\cdots+\left(g_{n}\right)^{1 / 2}\right]^{2}}{n^{2}} d \omega,
$$

where $d \omega$ is the area element, which can be expressed locally by the formula 


$$
d \omega=\left|\begin{array}{ll}
g_{11} & g_{12} \\
g_{12} & g_{22}
\end{array}\right|^{1 / 2} d u^{1} d u^{2}
$$

Making use of the inequality

$$
\left(\alpha_{1}+\cdots+\alpha_{n}\right)^{2} \leq n\left(\alpha_{1}^{2}+\cdots+\alpha_{n}^{2}\right),
$$

we have

$$
\begin{aligned}
A \bar{g}_{n} & \leq \iint n \frac{\left(g_{1}+\cdots+g_{n}\right)}{n^{2}} d \omega \\
& \leq \frac{\iint g_{1} d \omega+\cdots+\iint g_{n} d \omega}{n} \\
& \leq \frac{A_{g_{1}}+\cdots+A_{g_{n}}}{n} .
\end{aligned}
$$

By hypothesis, it follows that

$$
A_{\bar{g}_{n}} \leq A_{g_{1}}=\cdots=A_{g_{n}},
$$

which is ( $i)$.

The proof of (ii) follows from the definitions of the concepts concerned,

$$
\begin{aligned}
\int_{C} \bar{g}_{n}^{1 / 2} d s & =\int_{C} \frac{g_{1}^{1 / 2}+\cdots+g_{n}^{1 / 2}}{n} d s=\frac{\int_{C} g_{1}^{1 / 2} d s+\cdots+\int_{C} g_{n}^{1 / 2} d s}{n} \\
& \geq \frac{a_{g_{1}}+\cdots+a_{g_{n}}}{n}=a_{g_{1}}=\cdots=a_{g_{n}},
\end{aligned}
$$

the line integrals being extended along any curve $C$ of the family $F$. Hence

$$
\begin{aligned}
& a_{\bar{g}_{n}}=\text { g.l.b. }\left(\int_{C} \bar{g}_{n}^{1 / 2} d s\right) \geq a_{g_{1}}=\cdots=a_{g_{n}} \text {. } \\
& c \in F
\end{aligned}
$$

We shall now prove the following theorem, which characterizes the relationship between the two geometrical constants $A$ and $a$ in $M_{p^{2}}^{2}$. 
THEOREM 1. Let $M_{p^{2}}^{2}$ be the Riemannian manifold whose underlying topological space is a projective plane and whose metric is locally defined by

$$
d s^{2}=\sum_{i, k=1}^{2} g_{i k}\left(u_{1}, u_{2}\right) d u^{i} d u^{k}, \quad g_{i k}\left(u_{1}, u_{2}\right) \in C_{0} ;
$$

let $A$ be its area, and a the greatest lower bound of the lengths of all the closed curves not homotopic to zero on $\mathrm{M}_{\mathrm{p}^{2}}^{2}$; then

$$
A \geq \frac{2}{\pi} a^{2}
$$

Moreover, $2 / \pi$ is the best constant.

Proof. On account of the Weierstrass approximation theorem, it suffices to assume that the fundamental tensor $g_{i k}\left(u_{1}, u_{2}\right)$ is analytic. Then we can introduce, in the small, an isothermic coordinate system on $M_{p^{2}}^{2}$ so that the metric takes the form

$$
g^{*}\left(v_{1}, v_{2}\right)\left(d v_{1}^{2}+d v_{2}^{2}\right),
$$

where $g^{*}\left(v_{1}, v_{2}\right) \in C, g^{*}\left(v_{1}, v_{2}\right)>0$. We define the metric on the universal covering surface $S^{2}$ of the projective plane by a projection process. The Riemannian manifold $\mathrm{M}^{2}{ }_{\mathrm{S} 2}$ thus obtained is actually a Riemannian surface. According to the uniformization theorem, we can map $M_{S^{2}}^{2}$ onto the unit 2-sphere manifold $M_{U^{2}}^{2}$, and can arrange it in such a way that two diametrically opposite points of $U^{2}$ correspond to the same point of $M_{p^{2}}^{2}$. The metric has then the form

$$
d s^{2}=g(p) d \rho^{2} . \quad g(p)>0, g(p) \in C_{\omega} \text { for } p \in M_{U^{2}}^{2},
$$

where $d \rho^{2}$ is the line element of the unit sphere $U^{2}$ taken from the embedding 3-dimensional Euclidean space.

We remark that the area $A$ of $M_{p^{2}}^{2}$ is one half that of $M_{U^{2}}^{2}$.

Let us consider all the rotations $\sigma$ of the unit sphere $U^{2}$ about its center. All these rotations form a compact Lie group $G$. Applying the process of averaging over a compact Lie group, in this case the Hurwitz integration [2; 3 , p.188], we have

$$
\int_{G}\left[(g(p))^{1 / 2}\right]^{\sigma} \delta \sigma=[h(p)]^{1 / 2} .
$$


We shall show that $[h(p)]^{1 / 2}$ is invariant with respect to all the left translations

$$
\tau: \sigma \rightarrow \sigma^{\prime}=\tau \sigma, \tau, \sigma, \sigma^{\prime} \in G,
$$

and hence is a constant. In fact, let $\tau$ be any element of $G$; then, by definition,

$$
\left[(h(p))^{1 / 2}\right]^{\tau}=\int_{G}\left[(g(p))^{1 / 2}\right]^{\tau \sigma} \delta \sigma=\int_{G}\left[(g(p))^{1 / 2}\right]^{\tau \sigma} \delta \tau \sigma,
$$

since $\delta \sigma$ is invariant under all left translations. Therefore,

$$
[h(\tau p)]^{1 / 2}=\int_{G}\left[(g(p))^{1 / 2}\right]^{\lambda} d \lambda=[h(p)]^{1 / 2}, \quad \lambda=\tau \sigma \in G .
$$

As the group $G$ is transitive, $h^{1 / 2}$ is a constant.

Using $h d \rho^{2}$ instead of $g d \rho^{2}$ as the metric on the unit sphere $U^{2}$, we obtain a manifold with the spherical geometry. Preserving the metric $h d \rho^{2}$, and identifying the diametrical points on $U^{2}$, we get a manifold ${ }_{h} M^{2}{ }^{2}$, with the elliptic geometry. The two geometrical constants $A_{h}$ and $a_{h}$ can actually be evaluated:

$$
\begin{aligned}
A_{h} & =2 \pi h, \\
a_{h} & =\pi h^{1 / 2} .
\end{aligned}
$$

Hence

$$
A_{h}=\frac{2}{\pi} a_{h}^{2}
$$

It is clear that if $g(p)$ is subjected to a transformation $\sigma$ of $G$, the resulting metric $g^{\sigma}(p) d \rho^{2}$ is such that

$$
a_{g} \sigma=a_{g}
$$

and

$$
A_{g^{\sigma}}=A_{g}
$$

By approximating integrals by suitable sums and using Lemma 1, we easily obtain

$$
a_{h} \geq a_{g}
$$


and

$$
A_{h} \leq A_{g}
$$

Combining (4), (7), and (8), we have

$$
A_{g} \geq A_{h}=\frac{2}{\pi} a_{h}^{2} \geq \frac{2}{\pi} a_{g}^{2} .
$$

Dropping the unnecessary indices, we obtain the inequality

$$
A \geq \frac{2}{\pi} a^{2}
$$

That $2 / \pi$ is the best constant is evident, since we already have shown that the equality sign actually is attained when the metric is elliptic.

A slight generalization of Theorem 1 , referring to certain special Riemannian metrics on the $n$-dimensional projective space $P_{n}$, can be proved in a similar fashion, using Hölder's inequality

$$
\left(a_{1} b_{1}+\cdots+a_{m} b_{m}\right) \leq\left(a_{1}^{p}+\cdots+a_{m}^{p}\right)^{1 / p}\left(b_{1}^{q}+\cdots+b_{m}^{q}\right)^{1 / q}
$$

where $a_{i}, b_{i} \geq 0$ and $p, q>1$ such that $1 / p+1 / q=1$. The generalized theorem reads as follows:

THEOREM 2. Let $M_{p^{n}}^{n}$ be the Riemannian manifold whose underlying topological space is an $n$-dimensional projective space $P^{n}$, which we suppose represented by the unit $n$-sphere $U^{n}$ of the $(n+1)$-dimensional Euclidean space with identification of dianetrically opposite points $p$ and $p_{d}$, and whose metric can be represented in the form

$$
d s^{2}=g(p) d \rho^{2},
$$

where $g(p)>0, g(p) \in C_{0}, g(p)=g\left(p_{d}\right)$ for $p \in M_{p^{n}}^{n}$, and $d \rho^{2}$ is the lineelement of the n-sphere, taken from the embedding Euclidean space; let $V$ be its volume, and a the greatest lower bound of the lengths of all the closed curves which are not homotopic to zero on $M_{p^{n}}^{n}$; then

$$
V \geq \frac{\pi^{\frac{1-n}{2}}}{\Gamma\left(\frac{n+1}{2}\right)} a^{n}=k_{n} a^{n}
$$


Further, the above $k_{n}$ is the best constant.

The proof of Theorem 2 is left to the reader.

3. Riemannian manifold $M_{m}^{2}$, whose underlying topological space is a Moebius strip. Let $M_{m}^{2}$ be the Riemannian manifold whose underlying topological space is a Moebius strip and whose metric is locally defined by

$$
d s^{2}=\sum_{i \xi k=1}^{2} g_{i k}\left(u_{1}, u_{2}\right) d u^{i} d u^{k}, \quad g_{i k} \in C_{0} .
$$

By Weierstrass' approximation theorem, it suffices to assume that $g_{i k} \in C_{\omega}$. After introduction of the isothermic coordinate system, the metric takes the form

$$
d s^{2}=g^{*}(u, v)\left(d u^{2}+d v^{2}\right),
$$

where $g^{*}(u, v) \in C_{\omega}$ and $g^{*}(u, v)>0$. We define the metric $d s^{-2}$ on the universal covering surface of the Moebius strip by a projection process: $d s^{2}=d \bar{s}^{2}$; that is, the metric is invariant under the fundamental group of the Moebius strip. The covering manifold of $M_{m}^{2}$ thus obtained is actually a simply connected Riemann surface. According to the uniformization theorem, we can map it conformally onto a strip

$$
S:\left\{\begin{array}{l}
-\beta<y<\beta \\
-\infty<x<\infty
\end{array}\right.
$$

of the $(x, y)$-plane. The fundamental group $\Gamma$ appears then in the form:

$$
\sigma:\left\{\begin{array}{l}
x^{\prime}=x+n(2 \alpha), \\
y^{\prime}=(-1)^{n} y
\end{array} \quad(n=0, \pm 1, \pm 2, \cdots) .\right.
$$

The given manifold $M_{m}^{2}$ is mapped isogonally onto the fundamental region

$$
R:\left\{\begin{array}{l}
-\alpha \leq x<\alpha \\
-\beta<y<\beta
\end{array}\right.
$$

with a metric of the form

$$
d s^{2}=g(x, y)\left(d x^{2}+d y^{2}\right),
$$


where $g(x, y)>0$ and $g(x, y) \in C_{\omega}$. Moreover,

$$
g\left(x+n(2 \alpha),(-1)^{n} y\right)=g(x, y) \text {. }
$$

We are now in a position to prove the following theorem, which connects the two geometrical constants $A$ and $a$ in $M_{m}^{2}$.

THEOREM 3. Let $M_{m}^{2}$ be the Riemannian manifold whose underlying topological space is a Moebius strip and whose metric is locally defined by

$$
d s^{2}=\sum_{i, k=1}^{2} g_{i k}\left(u_{1}, u_{2}\right) d u^{i} d u^{k}, \quad g_{i k} \in C_{0}
$$

let $A$ be its area, and a the greatest lower bound of the lengths of all the closed curves which are not homotopic to zero on $M_{m}^{2}$; then we have

$$
A \geq \frac{2}{\pi} \cdot \frac{e^{\beta \pi / \alpha}-1}{e^{\beta \pi / a+1}} \cdot a^{2}=k_{a \beta} a^{2}
$$

where $2 \alpha$ and $2 \beta$ are the Euclidean lengths of the sides of the fundamental region $R$ of the Moebius strip. Moreover, the above constant $k_{a \beta}$ is best for a given ratio $\beta / \alpha$.

Proof. Let us consider the continuous group

$$
H:\left\{\begin{array}{l}
x^{\prime}=x+c \\
y^{\prime}=y
\end{array}\right.
$$

$c$ being real $(\bmod 4 \alpha) ; H$ consists of conformal transformations of the Moebius strip onto itself. It is evident that every two points which are equivalent under $\Gamma$ remain equivalent under $\Gamma$ after being operated on by elements of $H$. Defining the mean value, $[\bar{g}(y)]^{1 / 2}$, of $[g(x+c, y)]^{1 / 2}$ by the formula

$$
\frac{1}{4 \alpha} \int_{0}^{4 a}[g(x+c, y)]^{1 / 2} d c=[\bar{g}(y)]^{1 / 2}
$$

we can prove, by a method similar to that in the former cases, that

$$
\begin{aligned}
& \bar{A} \leq A, \\
& \bar{a} \geq a,
\end{aligned}
$$


where $\bar{A}$ and $\bar{a}$ have the same meaning as $A$ and $a$ except that we use the metric $\bar{g}(y)\left(d x^{2}+d y^{2}\right)$ instead of the general one. Moreover, from the invariance of $g(x, y)$ under the group $\Gamma$, it follows immediately that

$$
\bar{g}(y)=\bar{g}(-y) \text {. }
$$

We now shall consider the same problem with the simple metric

$$
d s^{2}=g(y)\left(d x^{2}+d y^{2}\right),
$$

where $g(-y)=g(y)>0$. Noting (9), we see that if the best inequality is found for such a $g(y)$, it is also found for all $g(x, y)$, and hence our problem is solved.

We are now going to determine a special positive, even, and for nonnegative $y$ monotonically decreasing function $g(y)$ such that a family $F^{*}$ of closed geodesic lines through the origin and not homotopic to zero on $M_{m}^{2}$ can be defined in terms of it.

Let us first establish a differential equation for such $g(y)$. Putting

$$
d s=\left[g(y)\left(1+x^{\prime 2}\right)\right]^{1 / 2} d y \quad\left(x^{\prime}=\frac{d x}{d y}\right),
$$

we know that the equation for the extremals is

$$
\frac{d}{d y}\left\{x^{\prime}\left[\frac{g(y)}{1+x^{\prime 2}}\right]^{1 / 2}\right\}=0 .
$$

Solving this equation, we have

$$
x=\int_{0}^{y} \frac{c d \eta}{\left[g(\eta)-c^{2}\right]^{1 / 2}}+k .
$$

Since the geodesics under consideration have to go through the origin, the constant $k$ has to be zero, and hence the equation becomes

$$
x=\int_{0}^{y} \frac{c d \eta}{\left[g(\eta)-c^{2}\right]^{1 / 2}} .
$$

The condition that the geodesics of the family be closed and not homotopic to zero requires that

$$
\left.\frac{d y}{d x}\right|_{(\alpha, \tau)}=0
$$


for $-\beta<\tau<\beta$; that is,

$$
[g(\tau)]^{1 / 2}=c
$$

for $-\beta<\tau<\beta$. Hence we have

$$
\alpha=\int_{0}^{\tau}\left[\frac{g(\tau)}{g(\eta)-g(\tau)}\right]^{1 / 2} d \eta, \quad 0 \leq \tau<\beta .
$$

For simplicity, let us normalize $g(y)$ so that $g(0)=1$. Since $g(y)$ is supposed to be monotonically decreasing for nonnegative $y$, we can put

$$
\begin{aligned}
& 1-g(\eta)=t, \\
& 1-g(\tau)=\omega,
\end{aligned}
$$

and have

$$
\begin{gathered}
\omega-t=g(\eta)-g(\tau) \\
d \eta=-\frac{d t}{g^{\prime}(\eta)} .
\end{gathered}
$$

Then equation (10) takes the form

$$
\frac{-\alpha}{[1-\omega]^{1 / 2}}=\int_{0}^{\omega} \frac{1}{g^{\prime}(\eta)} \cdot \frac{d t}{[\omega-t]^{1 / 2}}
$$

This is an Abel integral equation. According to the formula (cf. [1, p.484]) for the solution of such an equation,

$$
f(x)=\int_{0}^{x} \frac{y(t) d t}{(x-t)^{1 / 2}}
$$

we have

$$
y(t)=\frac{1}{\pi}\left[\frac{f(0)}{t^{1 / 2}}+\int_{0}^{t} \frac{f^{\prime}(z) d z}{(t-z)^{1 / 2}}\right]
$$

and, in our case,

$$
\frac{1}{g^{\prime}(\eta)}=-\frac{\alpha}{\pi}\left[\frac{1}{t^{1 / 2}}+\frac{1}{2} \int_{0}^{t} \frac{d z}{(1-z)^{3 / 2}(t-z)^{1 / 2}}\right]
$$


$=-\frac{\alpha}{\pi}\left[\frac{1}{t^{1 / 2}}+\frac{t^{1 / 2}}{1-t}\right]=-\frac{\alpha}{\pi} \cdot \frac{1}{(1-t) t^{1 / 2}}=-\frac{\alpha}{\pi} \cdot \frac{1}{g(\eta)[1-g(\eta)]^{1 / 2}}$

Thus we have established the differential equation for $g(y)$,

$$
\frac{d g}{d y}=-\frac{\pi}{\alpha} g(1-g)^{1 / 2} \text {. }
$$

The general solution of (13) is found to be

$$
\log \frac{1+(1-g)^{1 / 2}}{1-(1-g)^{1 / 2}}=\frac{\pi}{\alpha} y+k .
$$

When $y=0$, we have $[1-g(0)]^{1 / 2}=0$ and hence $k=0$. Therefore,

$$
\log \frac{1+(1-g)^{1 / 2}}{1-(1-g)^{1 / 2}}=\frac{\pi}{\alpha} y .
$$

An explicit expression for $g(y)$ is as follows:

$$
g(y)=\frac{4 e^{\pi y / a}}{\left(1+e^{\pi y / a}\right)^{2}}
$$

for $-\beta<y<\beta$.

From the explicit expression (14) for $g(y)$, every property of $g(y)$ we assumed at the beginning is verified. It is a positive, even, and monotonic decreasing function for $y \geq 0$. Moreover, $g(y) \rightarrow 0$ as $y \rightarrow \infty$ and $g^{\prime}(0)=0$. Such a $g(y)$, with those properties just mentioned and defining the family $F^{*}$ of the closed geodesics through $(0,0)$ and not homotopic to zero, is distinguished. Let us denote it by $g_{0}(y)$; that is, $g_{0}(y)$ is defined by either (13) or (14).

We are now in a position to establish the inequality in question for a positive, even, analytic function $g(y)$. By the definition of $a$, we have

$$
2 \int_{0}^{\tau}\left[g(y) \cdot\left(1+x^{\prime 2}\right)\right]^{1 / 2} d y=\varphi(\tau) \geq a
$$

for a closed curve, not homotopic to zero, on $M_{m}^{2}$. By taking this curve as one of $F^{*}$, we obtain 


$$
\left(1+x^{\prime 2}\right)^{1 / 2}=\left[\frac{g_{0}(y)}{g_{0}(y)-g_{0}(\tau)}\right]^{1 / 2} .
$$

Hence,

$$
\int_{0}^{\tau}\left[\frac{g(y) g_{0}(y)}{g_{0}(y)-g_{0}(\tau)}\right]^{1 / 2} d y=\frac{\varphi(\tau)}{2} \geq \frac{a}{2}
$$

This equation can be put into a more suitable form by setting

$$
\begin{aligned}
& 1-g_{0}(y)=t, \\
& 1-g_{0}(\tau)=\omega .
\end{aligned}
$$

Then we have

$$
\begin{aligned}
\omega-t & =g_{0}(y)-g_{0}(\tau), \\
d y & =-\frac{d t}{g_{0}^{\prime}(y)}, \\
\tau & =g_{0}^{-1}(1-\omega) .
\end{aligned}
$$

Equation (15) then takes the form

$$
\int_{0}^{\omega}\left[\frac{g(y) g_{0}(y)}{\omega-t}\right]^{1 / 2} \frac{d t}{g_{0}^{\prime} \cdot(y)}=\frac{\varphi}{2}\left[g_{0}^{-1}(1-\omega)\right] .
$$

In formula (12), we have

Solving (16), we obtain

$$
\begin{aligned}
& f(z)=-\frac{\varphi}{2}\left[g_{0}^{-1}(1-z)\right] \\
& f(0)=-\frac{\varphi(0)}{2} \\
& f^{\prime}(z)=\frac{1}{2} \cdot \frac{\varphi^{\prime}\left(g_{0}^{-1}(1-z)\right)}{g_{0}^{\prime}\left(g_{0}^{-1}(1-z)\right)} .
\end{aligned}
$$

$$
\frac{\left[g(y) g_{0}(y)\right]^{1 / 2}}{g_{0}^{\prime} \cdot(y)}=\frac{1}{\pi}\left[-\frac{\varphi(0)}{2 t^{1 / 2}}+\frac{1}{2} \int_{0}^{t} \frac{\varphi^{\prime}\left(g_{0}^{-1}(1-z)\right)}{g_{0}^{\prime}\left(g_{0}^{-1}(1-z)\right)} \cdot \frac{d z}{(t-z)^{1 / 2}}\right]
$$


Putting

$$
\begin{aligned}
& g_{0}^{-1}(1-z)=u, \\
& g_{0}^{-1}(1-t)=y,
\end{aligned}
$$

we have

$$
\frac{\left[g(y) g_{0}(y)\right]^{1 / 2}}{g_{0}^{\prime}(y)}=-\frac{\varphi(0)}{2 \pi\left[1-g_{0}(y)\right]^{1 / 2}}-\frac{1}{2 \pi} \int_{0}^{y} \frac{\varphi^{\prime}(u) d u}{\left[g_{0}(u)-g_{0}(y)\right]^{1 / 2}},
$$

or

$$
\left[g(y) g_{0}(y)\right]^{1 / 2}=-\frac{\varphi(0) g_{0}^{\prime}(y)}{2 \pi\left[1-g_{0}(y)\right]^{1 / 2}}-\frac{1}{2 \pi} g_{0}^{\prime} \cdot(y) \int_{0}^{y} \frac{\varphi^{\prime}(u) d u}{\left[g_{0}(u)-g_{0}(y)\right]^{1 / 2}} .
$$

Integrating from 0 to $\beta$, we get

$$
\begin{aligned}
\int_{0}^{\beta}\left[g(y) g_{0}(y)\right]^{1 / 2} d y=- & \frac{\varphi(0)}{2 \pi} \int_{0}^{\beta} \frac{g_{0}^{\prime}(y)}{\left[1-g_{0}(y)\right]^{1 / 2} d y} \\
& -\frac{1}{2 \pi} \int_{0}^{\beta} g_{0}^{\prime}(y)\left\{\int_{0}^{y} \frac{\varphi^{\prime}(u) d u}{\left[g_{0}(u)-g_{0}(y)\right]^{1 / 2}}\right\} d y \\
= & \frac{\varphi(0)}{\pi}\left[1-g_{0}(\beta)\right]^{1 / 2} \\
& -\frac{1}{2 \pi} \int_{0}^{\beta} \varphi^{\prime}(u)\left\{\int_{u}^{\beta} \frac{g^{\prime}(y) d y}{\left[g_{0}(u)-g_{0}(y)\right]^{1 / 2}}\right\} d u \\
= & \frac{\varphi(0)}{\pi}\left[1-g_{0}(\beta)\right]^{1 / 2} \\
& +\frac{1}{\pi} \int_{0}^{\beta} \varphi^{\prime}(u)\left[g_{0}(u)-g_{0}(\beta)\right]^{1 / 2} d u \\
= & -\frac{1}{\pi} \int_{0}^{\beta} \varphi^{\prime}(u)\left\{\frac{d}{d u}\left[g_{0}(u)-g_{0}(\beta)\right]^{1 / 2}\right\} d u
\end{aligned}
$$




$$
=\frac{1}{\pi} \int_{0}^{\beta} \varphi(u)\left\{-\frac{d}{d u}\left[g_{0}(u)-g_{0}(\beta)\right]^{1 / 2}\right\} d u .
$$

We remark that $-d\left[g_{0}(u)-g_{0}(\beta)\right]^{1 / 2} / d u \geq 0$. Squaring and applying Schwarz's inequality, we have

$$
\begin{aligned}
& \left\{\int_{0}^{\beta}\left[g(y) g_{0}(y)\right]^{1 / 2} d y\right\}^{2} \\
& =\frac{1}{\pi^{2}}\left\{\int_{0}^{\beta} \varphi(u)\left(-\frac{d}{d u}\left[g_{0}(u)-g_{0}(\beta)\right]^{1 / 2}\right) d u\right\}^{2}, \\
& \int_{0}^{\beta} g(y) d y \cdot \int_{0}^{\beta} g_{0}(y) d y \geq \frac{a^{2}}{\pi^{2}}\left[1-g_{0}(\beta)\right] .
\end{aligned}
$$

The equality sign holds when $g=g_{0}$ due to a converse part of the theorem on Schwarz's inequality. Then we have

$$
\int_{0}^{\beta} g(y) d y \geq \frac{a^{2}}{\pi^{2}} \cdot \frac{1-g_{0}(\beta)}{\int_{0}^{\beta} g_{0}(y) d y} .
$$

From (14), we can easily compute

$$
\begin{gathered}
1-g_{0}(\beta)=\left(\frac{e^{\pi \beta / \alpha}-1}{e^{\pi \beta / \alpha}+1}\right)^{2}=\frac{\pi^{2}}{4} k_{\alpha \beta}^{2} \\
\int_{0}^{\beta} g_{0}(y) d y=\frac{2 \alpha}{\pi} \cdot \frac{e^{\pi \beta / \alpha}-1}{e^{\pi \beta / \alpha}+1}=k_{\alpha \beta} \alpha .
\end{gathered}
$$

Combining (17), (18), and (19), we obtain

$$
\int_{0}^{\beta} g(y) d y \geq k_{\alpha \beta} \frac{a^{2}}{4 \alpha} .
$$


We remark that the equality sign in (20) holds for $g=g_{0}$. In fact, in this case, it can easily be proved that $a=2 \alpha$ by using the Weierstrass theory of extremal fields. From (19), we have

$$
\int_{0}^{\beta} g_{0}(y) d y=\alpha k_{\alpha \beta}=k_{\alpha \beta} \frac{a^{2}}{4 \alpha} .
$$

Combining the definition of area and (20), we obtain

$$
A=4 \alpha \int_{0}^{\beta} g(y) d y \geq k_{a \beta} a^{2} .
$$

By (21), we know that

$$
A=k_{\alpha \beta} a^{2}
$$

for $g=g_{0}$. This shows that our $k_{\alpha \beta}$ is the best constant.

3. Added in proof. In a course on Riemannian Geometry given at Syracuse University in 1949, Professor C. Loewner proved the inequality $A \geq 31 / 2 a^{2} / 2$ for the case of $M_{t}^{2}$, the Riemannian manifold whose underlying topological space torus. The present investigation originates from this idea and has a similar method of treatment.

\section{REFERENCES}

1. Ph. Frank und R. v. Mises, Die Differential- und Integralgleichungen der Mechanik und Physik, Erster Teil, New York, 1943.

2. A. Hurwitz, Über die Erzeugung der Invarianten durch Integration, Nachr. Akad. Wiss. Göttingen. Math.-Phys. Kl. Math.-Phys.-Chem. Abt. 1897, 71-90.

3. H. Weyl, Classical Groups, Their Invariants and Representations, Princeton University Press, 1939.

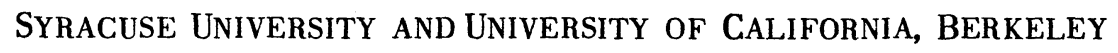





\section{EDITORS}

HERBERT BUSEMANN

University of Southern California

Los Angeles 7, California
R. M. ROBINSON

University of California

Berkeley 4, California

E. F. BECKENBACH, Managing Editor

University of California

Los Angeles 24, California

\section{ASSOCIATE EDITORS}

\author{
R. P. DILWORTH \\ HERBERT FEDERER \\ MARSHALL HALL
}

P. R. HALMOS

HEINZ HOPF

R. D. JAMES

\author{
BØRGE JESSEN \\ PAUL LÉVY \\ GEORGE PÓLYA
}

J. J. STOKER
E. G. STRAUS

KÖSAKU YOSIDA

\section{SPONSORS}

UNIVERSITY OF BRITISH COLUMBIA

CALIFORNLA INSTITUTE OF TECHNOLOGY

UNIVERSITY OF CALIFORNIA, BERKELLEY

UNIVERSITY OF CALIFORNIA, DAVIS

UNIVERSITY OF CALIFORNIA, LOS ANGELES

UNIVERSTTY OF CALIFORNIA, SANTA BARBARA

OREGON STATE COLLEGE

UNIVERSITY OF OREGON
UNIVERSETY OF SOUTHERN CALIFORNIA STANFORD UNIVERSITY

WASHINGTON STATE COLLEGE UNIVERSTTY OF WASHINGTON

AMERICAN MATHEMATICAL SOCIETY NATIONAL BUREAU OF STANDARDS, INSTITUTE FOR NUMERICAL ANALYSIS

Vari-Type Composition by

Elaine Barth

Delores Gilbertson

With the cooperation of

E. F. Beckenbach

E. G. Straus

Printed in the United States of America by

Edwards Brothers, Inc., Ann Arbor, Michigan

UNIVERSITY OF CALIFORNIA PRESS * BERKELEY AND LOS ANGELES

COPYRIGHT 1952 BY PACIFIC JOURNAL OF MATHEMATICS 


\section{Pacific Journal of Mathematics}

\section{Vol. 2, No. $1 \quad$ January, 1952}

Tom M. (Mike) Apostol, Theorems on generalized Dedekind sums ........ 1

Tom M. (Mike) Apostol, Addendum to 'On the Lerch zeta function' ........ 10

Richard Arens, Extension of functions on fully normal spaces ........... 11

John E. Maxfield, A short proof of Pillai's theorem on normal numbers.... 23

Charles B. Morrey, Quasi-convexity and the lower semicontinuity of multiple integrals................................ 25

P. M. Pu, Some inequalities in certain nonorientable Riemannian manifolds....................................... 55

Paul V. Reichelderfer, On the barycentric homomorphism in a singular

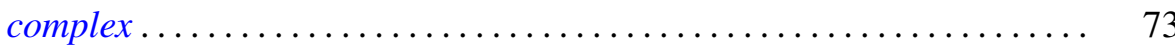

A. H. Stone, Incidence relations in multicoherent spaces. III ........... 99 